\title{
What Does Consciousness Have to Do With It? Quality of Life in Patients With Disorders of Consciousness
}

\section{Lily Frank \& Michał Klincewicz}

To cite this article: Lily Frank \& Michał Klincewicz (2016) What Does Consciousness Have to Do With It? Quality of Life in Patients With Disorders of Consciousness, AJOB Neuroscience, 7:1, 50-52

To link to this article: http://dx.doi.org/10.1080/21507740.2016.1150919

曲 Published online: 04 Apr 2016.

Submit your article to this journal $\pi$

Q View related articles ¿

View Crossmark data 
Nagel, T. 1974. What it's like to be a bat. Philosophical Review 83:435-50.

Peterson, A., Cruse, D., Naci, L., Weijer, C., and Owen, A. M. 2015. Risk, diagnostic error, and the clinical science of consciousness. Neuroimage: Clinical 7:588-97.
Sherman, S. J. 1980. On the self-erasing nature of errors of prediction. Journal of Personality and Social Psychology 39: $211-21$

Wittgenstein, L. 1953. Philosophical investigations. PI 23. Hoboken, NJ: Blackwell.

\title{
What Does Consciousness Have to Do With It? Quality of Life in Patients With Disorders of Consciousness
}

\author{
Lily Frank, Eindhoven University of Technology \\ Michal Klincewicz, Jagiellonian University
}

L. Syd M Johnson's article argues for caution in the clinical practices surrounding prognostication for patients with disorders of consciousness (DOC). The epistemic limitations of current neuroscience and the pressures that surrogates and physicians face when making decisions about continuing or withdrawing treatment create an urgency for further research into how to understand, measure, and manage quality of life in these patients. Because of this, the relationship between conscious experience and other psychological functions that would inform such a research program needs to be carefully examined.

The extant literature on the nature of conscious experience shows that it is highly controversial what role, if any, consciousness plays in psychological functioning. Consider reasoning and the thoughts and beliefs that it purportedly involves. These psychological states bear intentional content-that is, they are about something in the same way that sentences are about something. But whatever the functional role of intentional content in reasoning is, it is connected to that aboutness and not to consciousness (Rosenthal 2008). A thought may have the content "Tuesdays come just before Wednesdays of the same week." This thought, together with another thought with the content "today is Tuesday," licenses the inference to another thought with the content "tomorrow is Wednesday" irrespective of whether it is conscious. The same is true of beliefs. Consider a belief that "Tuesdays come just before Wednesdays of the same week." This belief does not explicitly figure in inferences about weekend plans, even though it implicitly informs every such inference. Perceptual states primarily allow us to make discriminations in the environment, and again, this is independent of whether they are accompanied by conscious awareness; perception can occur without awareness (Prinz
2015). Finally, emotional states are notorious for their influence outside of consciousness (Wyer 2014; Bargh 2007). What all of this amounts to is that it is at least controversial whether consciousness plays a significant (or any) role in the functionings of emotions, perceptions, and intentional states, such as thoughts and beliefs. Put bluntly, consciousness might not matter that much as far as the rest of our psychology is concerned (Rosenthal 2008).

If consciousness is not that important to other mental functions, then this raises the question of whether it should be important in the assessment of quality of life and decisions about treatment for patients with DOC. Perhaps when doctors use the word "consciousness" they refer to an amalgam of other psychological capacities, such as emotions, thoughts, and so on. If that is so, then they are simply using the word in a way that obscures the connection to quality-of-life assessment. Another possibility is that "consciousness" in this context refers not to a state that is phenomenally conscious, but to the whole organism that is in a state that contrasts with being asleep or in a coma (Rosenthal 1986; Block 1995). But this cannot be the type of consciousness to which DOC refers, since there is a clear clinical distinction between states like unresponsive wakefulness syndrome (formerly the vegetative state), in which the patient exhibits demarcated sleep-wake cycles, and states like the coma where this is not the case.

Although quality of life is a contested concept that is theorized about in economics, philosophy, and psychology, it is uncontroversial that emotions, perceptions, thoughts, and beliefs are a part of a person's quality of life. And it is these types of mental states that play an important role in nearly every form of quality-of-life assessment. Probably the most robust quantitative instruments for this purpose have been developed in the field of medicine and

Address correspondence to Lily Frank, PhD, Assistant Professor, Philosophy and Ethics, Innovation Science, Eindhoven University of Technology, 5612 AZ, Eindhoven, The Netherlands. E-mail: L.e.frank@tue.nl 
health economics. These health-related quality of life (HRQOL) measures are used primarily for the purposes of making national and international health policies, specifically, deciding to which interventions, medicines, and devices scarce financial resources should be allocated. Measurements like quality adjusted life years (QALYS) allow for comparisons to be made across a wide range of diseases, conditions, and patient groups. HRQOL measurements take into account the "physical, psychological, and social domains of health, seen as distinct areas (or domains) that are influenced by a person's experiences, beliefs, expectations, and perceptions" (Testa and Simonson 1996,55$)$.

There are many different instruments for measuring HRQOL: some for specific diseases and conditions, and others for particular domains, like pain or depression, as well as generic instruments. These instruments can be adapted to assess the quality of life of patients with DOC, but then one is immediately presented with the problem that they rely on gathering data through self-reports, that is, asking patients things like "How much time during the past month have you felt downhearted and depressed?" (SF-12). There is a body of research on the HRQOL of people with cognitive disabilities and dementia. These assessments usually rely on one or a combination of patient selfreport (to the extent that it is possible), observation of patient behavior, and proxy reports by caretakers or family members (Logsdon et al. 2002). For patients with DOC none of these methods can be straightforwardly implemented. Some attempts have also been made to develop QOL measures for neonates in the intensive care unit. They share with patients with DOCs an inability to issue self-reports. Assessments of neonates' quality of life by proxy are also unreliable because of the likelihood that the proxy's assessment reflects his or her own QOL, rather than the patient's (Boss et al. 2012). Another feature these two groups share is that projected future QOL, rather than present QOL, is often taken to be the more relevant factor when making decisions about treatment (Boss et al. 2012, 902). Boss and colleagues recommend using a combination of physiological data, proxy reports, and dimensions that are specifically thought to be relevant to the well-being of infants, such as level of light exposure, skin contact, and invasive procedures, to begin to understand HRQOL in neonates. Just as for infants or the cognitively impaired, HRQOL assessment will have to be specifically tailored to patients with DOC and will likely have to involve a range of types of measures. Johnson's article gives us several reasons to think that the development of such measures is crucial. First, medicine does not have a very high success rate at predicting recovery from or improvement in disorders of consciousness, and standard practice is to err on the side of a pessimistic prediction about recovery and life prospects. As Johnson suggests, giving these patients more time to stabilize and to watch for changes and improvements may be prudent advice for the surrogate decision makers for these patients. If it does indeed become standard practice to adopt this wait and see approach, then there is an even higher obligation on the part of medicine to strive to be able to give surrogates insights into the current quality of life of the patients, as well as their predicted future quality of life. In order to weigh the costs and benefits of waiting for recovery for an additional several months, surrogates require some information, at the very least, that the patient is not in severe pain or mental distress or that these symptoms are being controlled. Of course, this is putting aside considerations of cost and resource allocation, which are beyond the scope of this short commentary. Another reason why it is crucial to develop QOL measures for these patients is that standards of care for patients with a DOC may have to reflect a concern not only for the potential recovery of consciousness, but for the maintenance of other mental faculties, which are more closely tied to their quality of life.

In conclusion, patients with DOC, even while completely unconscious, may nonetheless have psychological states relevant to assessment of their quality of life at that time. There are theoretical and empirical reasons to think that this is the case. First, for all the reasons we have mentioned, we can expect psychological states to sometimes occur without consciousness. Second, we know that some patients with DOC still retain some brain function in areas that are thought to be involved with sensations, emotions, and even reasoning, even if they have little to no ability to communicate or express these mental states (Monti et al. 2010).

\section{REFERENCES}

Bargh, J. A. 2007. Social psychology and the unconscious: The automaticity of higher mental processes. New York, NY: Psychology Press.

Block, N. 1995. How many concepts of consciousness? Behavioral and Brain Sciences 18 (02):272-287.

Boss, R. D., H. I. Kinsman, and P. K. Donohue. 2012. Healthrelated quality of life for infants in the neonatal intensive care unit. Journal of Perinatology 32(12): 901-6.

Johnson, L. S. M. 2016. Inference and inductive risk in disorders of consciousness. AJOB Neuroscience 7(1): 35-43.

Logsdon, R. G., L. E. Gibbons, S. M. McCurry, and L. Teri. 2002. Assessing quality of life in older adults with cognitive impairment. Psychosomatic Medicine 64(3): 510-19.

Monti, M. M., A. Vanhaudenhuyse, M. R. Coleman, M. Boly, J. D. Pickard, L. Tshibanda, A. M. Owen, and S. Laureys. 2010. Willful modulation of brain activity in disorders of consciousness. New England Journal of Medicine 362(7): 57989.

Prinz, J. J. 2015. Unconscious perception. In The Oxford handbook of the philosophy of perception, ed. M. Matthen, 371. Oxford, UK: Oxford University Press.

Rosenthal, D. M. 2008. Consciousness and its function. Neuropsychologia 46(3): 829-40.

Rosenthal, D. M. 1986. Two concepts of consciousness. Philosophical Studies 49(3): 329-59. 
Testa, M. A., and D. C. Simonson. 1996. Assessment of qualityof-life outcomes. New England Journal of Medicine 334(13): 835-840.
Wyer, R. S. 2014. The automaticity of everyday life: Advances in social cognition, Vol. 10. New York, NY: Psychology Press.

\title{
Deferring to the Best Inferrer? Ethically Inferring on Behalf of Patients With Disorders of Consciousness
}

\author{
Valerye M. Milleson, Albany Medical College
}

While L. Syd Johnson has elucidated some very real problems with current inferences in and the corresponding medical management of patients with disorders of consciousness (DOCs) and offered a worthwhile preliminary set of suggested clinical responses to these problems, from the perspective of one currently working in clinical ethics consultation these suggestions do not sufficiently address the concerns of managing inductive risks in decision making regarding patients with DOCs. In this commentary I discuss what I believe are two particularly problematic gaps in Johnson's recommendations and offer a tentative account of some additional measures that may be useful in moving forward with managing inductive risks in decision making for these patients.

One major area of concern is the author's apparent reliance on surrogate decision making for these patients. At present, the two primary standards used by surrogates when deciding on behalf of patients lacking decision-making capacity are substituted judgment and best interests, both of which present challenges in the case of patients with DOCs. In brief, when a surrogate applies the substituted judgment standard, he or she "choos[es] as the incompetent individual would choose in the circumstances were he or she competent" (Buchanan and Brock 1990, 112). In our scenario, then, a surrogate would be asked to decide what the patient with DOC would want in terms of treatment or nontreatment if presently able to understand his or her medical situation and express those preferences. The first challenging aspect of this has to do with what it would mean to understand-and indeed to have a preference about-the current medical situation. As Johnson has pointed out, there is a high degree of uncertainty with respect to providing accurate medical prognostication in these cases, leading to inferences about not only the sort of DOC a patient has (including the corresponding likelihood of recovery), but also whether or not in that particular DOC the patient can be or is experiencing suffering. What, then, can the patient's preferences be about? On the face of it, they have to be preferences based on probabilities-but in our given situation the probabilities themselves are suspect. The uncertainty experienced in the case of patients with DOCs calls into question the possibility of there even being preferences other than the extremes of "any chance at recovery is worth proceeding with treatment" and "no chance at recovery is worth risking being 'stuck with life'" that can be meaningfully substituted on behalf of these patients. This problem is compounded by the many studies indicating that surrogates are often unable to accurately predict a patient's preferences for treatment or nontreatment in a given medical scenario, including scenarios where diagnostic and prognostic inferences are far more certain (Shalowitz, Garrett-Mayer, and Wendler 2006; Fagerlin et al. 2001). But for patients with DOCs, we are asking surrogates to make accurate inferences about a patient's preferences even when the medical inferences surrogates have to base them on are highly tenuous, leaving little assurance that the surrogate's decision will ultimately reflect the patient's preferences and thereby be "the option most likely to result in decisions that accord with the wishes, preferences, and values of the patients themselves" (Johnson 2016, 40).

The other main standard for surrogate decision making, the best interests standard, is no better at offering guidance in these situations. When a surrogate applies the best interests standard, he or she is asked to weigh the pros and cons of the available treatment options and determine which option is in the best interests of the patient. Here again, when considering what is in the best interests of a patient with DOC there will necessarily be a lot of inferences that are being made. Faced with such high degrees of variability and uncertainty, it is not clear how successful any of these inferences can or will be at helping surrogates determine a course of action that is actually in the best interest of the patient. If, as Johnson recommends, DOC patients are granted greater recovery time to allow for improved diagnostic and prognostic accuracy, these

Address correspondence to Valerye M. Milleson, Albany Medical College, Alden March Bioethics Institute, 47 New Scotland Avenue, MC 153, Albany, NY 12208, USA. E-mail: MillesV@mail.amc.edu 